\title{
Cutaneous odontogenic lesion: a frequently misdiagnosed entity
}

\author{
Payal Saxena ${ }^{1}$, Saurabh Kumar Gupta ${ }^{1}$, Pratibha Katiyar ${ }^{2}$, Shivam Kamthan ${ }^{3}$, \\ Parul Saxena ${ }^{4}$ \\ ${ }^{I}$ (Department Of Conservative Dentistry, Government College Of Dentistry, \\ MP Medical Science University, India) \\ ${ }^{2}$ (Department Of Prosthodontics, Career Dental College, Lucknow, India) \\ ${ }^{3}$ (Department Of Social And Preventive Medicine, Swami Vivekanand Subharti University, \\ Meerut, India) \\ ${ }^{4}$ (Department Of Anatomy, Lala Lajpat Rai Memorial Medical College, Meerut, India)
}

\begin{abstract}
Possible dental etiology is often overlooked in the patients with cutaneous odontogenic lesion. An accurate diagnosis requires interaction between physicians (especially dermatologists), surgeons, and dentists. Early recognition of the lesion facilitates prompt treatment, minimizes patient discomfort and esthetic problems, and reduces the possibility of further complications greatly. This article presents three case reports of cutaneous odontogenic lesion highlighting the importance of communication between medical and dental practitioners to avoid misdiagnosis.
\end{abstract}

Keywords: Dental fistula, Drainage, Endodontics, Esthetics dental, Etiology

\section{Introduction}

The diagnosis of a cutaneous lesion of dental origin can be challenging for several reasons. As specific dental symptoms usually are absent in these cases, patients typically first visit a physician for evaluation and treatment. It has been reported that about half of the patients undergo multiple surgeries and trials of antibiotics before definitive diagnosis [1]. Proper diagnosis can lead to simple and effective treatment consisting of removal of the infected pulp canal tissue, resulting in minimal cutaneous scarring [2].

The aim of the present article is to present the successful management of three cases with cutaneous lesions of dental origin two of which were earlier misdiagnosed as having cutaneous etiology. These reports also highlight the importance of communication between physicians and general dentistry practitioners in the evaluation of patients with head and neck lesions.

\subsection{Case 1}

\section{Case report}

A 21 year old female was referred to the authors with the chief complaint of a soft tissue overgrowth in the chin area. Her history revealed that she had developed a small growth on the skin of her chin three years back that slowly started enlarging in size. She has undergone multiple excisional surgeries, after a diagnosis of chronic suppurating lesion was made following histopatologocal examination, but every time the growth recurred after 3-4 months. She was then referred to the dental hospital for opinion regarding the same. Extraoral examination showed an elevated erythamatous non tender nodular lesion in submental region which had an indurated base (Fig. 1a). On palpating slight amount of pus discharge was evident. Intraorally, no vestibular swelling was observed, mandibular right central incisor (41) and left central incisor (31) were slightly tender on percussion. Vitality test was not responsive. An intraoral periapical (IOPA) radiograph of the concerned area was advised which showed periapical radiolucency in relation to 31 and 41 (Fig 1c). A diagnosis of chronic suppurative periradicular periodontitis, due to necrotic pulp, with cutaneous discharge was made. Root canal treatment of 31 and 41 was planned. Endodontic access tooth preparation was done. Working length determination, cleaning, shaping and proper debridement of the canals were carried out followed by intracanal medicament (calcium hydroxide) placement. Patient was recalled one week later. The growth had reduced in size and the teeth were asymptomatic (Fig. 1b). Obturation and sealing of the access cavity of the prepared teeth was done (Fig. 1d) and the patient again recalled 4 weeks later. On the recall appointment the healing of the lesion was almost complete except for a scar tissue which had formed there (Fig. 1e). The patient opted for a surgical aesthetic correction. On her follow-up visit after surgery the scar tissue was almost obliterated (Fig. 1f).

\subsection{Case 2}

A 18 year old female patient visited to the authors with the chief complaint of swelling and pus discharge from chin area since 8 days. Patient was apparently asymptomatic three years back when she suffered 
such swelling and pus discharge for the first time. She consulted a general physician who drained this area and the problem subsided for that time but later recurred again, repeating itself every 3-4 months. The physician then referred her to a dentist who did oral prophylaxis and instructed her oral hygiene measures. Finally, the patient came to our department with the same problem.

On extraoral examination, there was an ulcer with discharging sinus in submental area (Fig. 2a \& b). Intraorally, the mandibular incisors were non carious. Vitality test showed that 41 was non vital. IOPA radiographs revealed periapical pathology in right mandibular central incisor (Fig. 2c). Thus, a diagnosis of chronic apical periodontitis with pulpal necrosis was made and root canal treatment was planned with patient's consent and was performed as in case 1 (Fig. 2d). Patient was recalled after two weeks and satisfactory healing was observed (Fig. 2e \& f).

\subsection{Case 3}

A 30 year old male patient reported to our department with the chief complaint of protuberance in the chin area for the past one month and pus discharge from the same area after he shaved one day before. The patient was apparently asymptomatic two years back when he first noticed a swelling in the chin area for which he never consulted a medical or dental practitioner. He recollected that whenever he shaved, there was discharge in the area and the swelling subsided but recurred again later. On suggestion of a friend, he came to our department. Extraoral examination showed a nodular swelling with sinus in supramental area (Fig 3a \& b). Intraorally, the teeth were non carious and asymptomatic on percussion, slight discoloration was evident in relation to teeth number 31 and 42 (Fig. 3e). Vitality test was negative for the same teeth $(31 \& 42)$. IOPA showed periapical pathology in relation to teeth number 31 and 42(Fig. 3c). A diagnoses of chronic apical periodontitis with pulpal necrosis was made and root canal treatment was completed with patient's consent (Fig. 3d). A satisfactory healing was seen on recall visit after two weeks (Fig. 3f).

\section{Discussion}

The characteristic cutaneous lesion of odontogenic origin appears as an erythematous, soft nodule fixed to underlying structures. There is periodic drainage and crusting in some cases. Palpation of the surrounding tissue may produce pus, which supports the diagnosis[1,2]. The majority lesions arise on the chin, mandible, or submandibular area [3]. On the basis of clinical appearance, the differential diagnosis includes pyogenic granuloma, actinomycosis, thyroglossal duct cyst, branchial cleft cyst, furuncle, squamous cell carcinoma, and epidermal cyst [4]. Careful questioning of the patient about past symptoms may help identify a dental cause but symptoms may not be present in all cases. Intraoral examination is critical. Vitality testing of potentially causal teeth should be undertaken, non vital tooth may appear discolored as seen in case 3 (Fig. 3e). Radiographs will show a radiolucency at the apex of the infected tooth [1]. It has been suggested that computerized tomography may be superior to panoramic or intraoral radiographs [5]. Biopsy, if performed, will show nonspecific findings such as hyperplasia and chronic inflammation [2]. Once the correct diagnosis is made, root canal therapy or surgical extraction is the treatment of choice and antibiotics may be used as an adjunct to surgical therapy. The cutaneous lesion usually resolves in one to two weeks [1]. Depression and scar formation of the area are not uncommon and usually fade with time [6]. Surgical correction of the scar can be undertaken to provide better a cosmetic result [7] (as performed in case 1; Fig. 1f). Failure of a cutaneous sinus tract to heal after adequate root canal treatment or extraction requires further evaluation and biopsy.

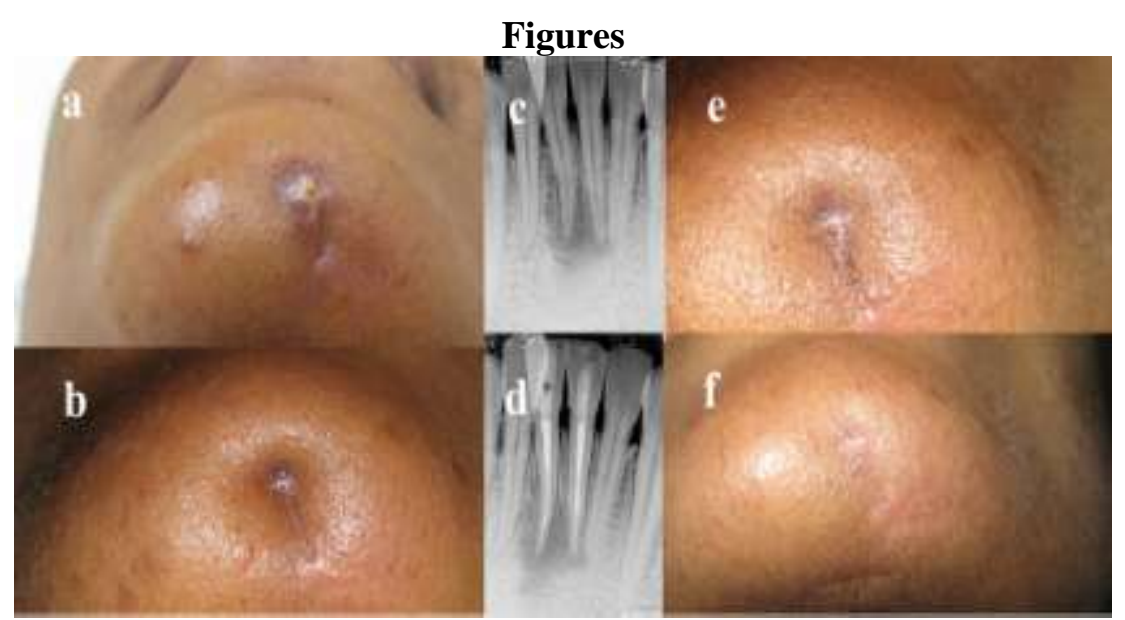

Figure 1 (a) Clinical examination revealed an erythematous nodular lesion in submental area; (b) reduction of growth on recall appointment after placing intracanal medicament; (c) pre operative radiograph; (d) post 
operative radiograph; (e) post operative photograph showing good healing except scar tissue; (f) complete healing after surgical aesthetic correction.

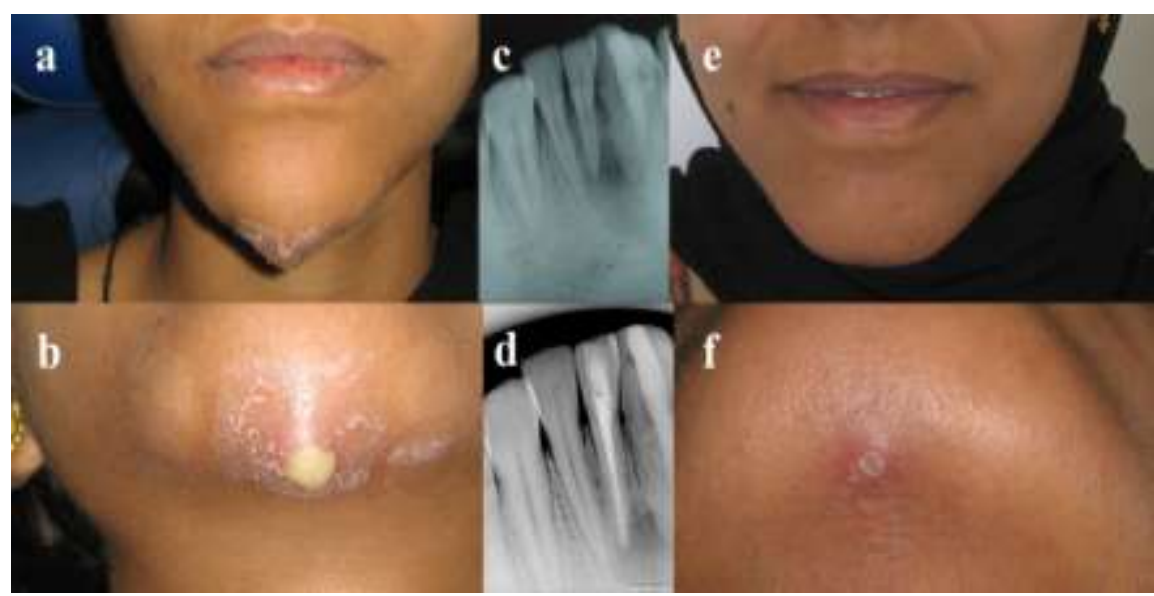

Figure 2 (a) \& (b) Clinical examination showed an ulcer with draining sinus in submental area; (c) pre operative radiograph; (d) post operative radiograph; (e) \& (f) post operative photographs showing satisfactory healing.

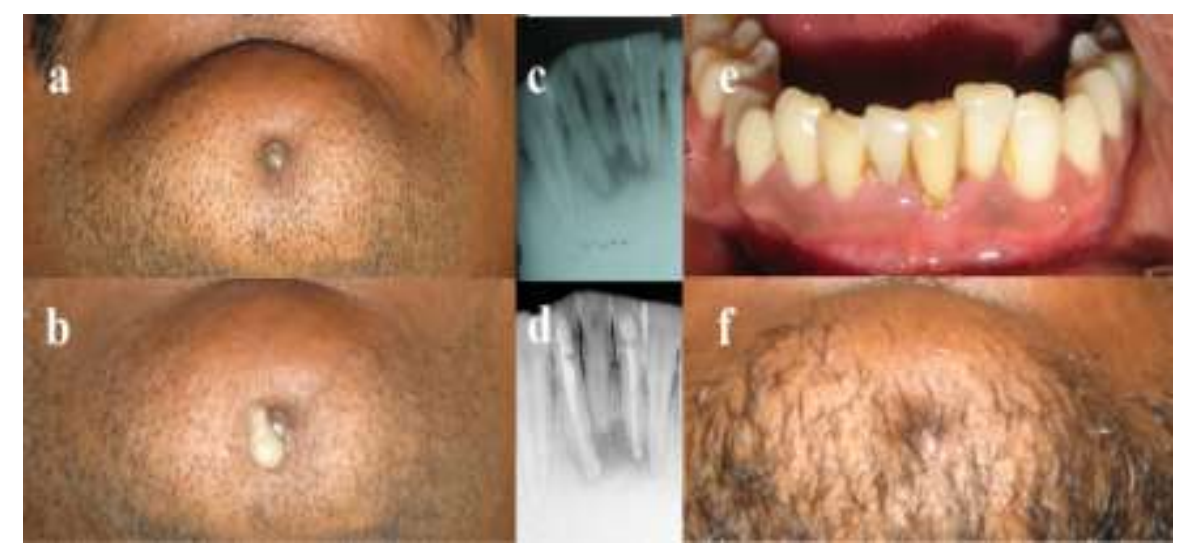

Figure 3 (a) Clinical examination showed nodular swelling with sinus in submental area; (b) on palpation, pus discharge was evident (c) pre operative radiograph; (d) post operative radiograph; (e) intraoral examination revealed slight discoloration of teeth number $31 \& 42$; (f) post operative photograph showing satisfactory healing.

\section{Conclusion}

In accordance to the above cases described, cutaneous lesions of dental origin are often misdiagnosed and inappropriately treated. Patient dissatisfaction following such diagnostic and therapeutic mishaps is reasonably high. Clinicians should be aware of the fact that any cutaneous lesion of the face and neck can be of dental origin and should seek communication and evaluation from concerned specialists and general dental practitioners.

\section{References}

[1]. Cantatore JL, Klein PA, Lieblich LM. Cutaneous dental sinus tract, a common misdiagnosis: a case report and review of the literature. Cutis 7, 2002, 264-267.

[2]. Stoll HL, Solomon HA. Cutaneous sinuses of dental origin. Journal of the American Medical Association, 184, 1963, 120-124.

[3]. Cioffi GA, Terezhalmy GT, Parlett HL. Cutaneous draining sinus tract: an odontogenic etiology. Journal of American Academy of Dermatology, 14, 1986, 94-100.

[4]. Held JL, Yunakov MJ, Barber RJ, et al. Cutaneous sinus of dental origin: a diagnosis requiring clinical and radiologic correlation. Cutis, 43, 1989, 22-24.

[5]. Bodner L, Bar-Ziv J. Cutaneous sinus tract of dental origin: imaging with a dental CT software programme. British Journal of Oral and Maxillofacial Surgery, 36, 1998, 311-313.

[6]. Gupta SK, Saxena P. Cutaneous manifestation of odontogenic infection misdiagnosed as having dermatologic etiology: a report of two cases. Quintessence International, 42, 2011, 455-458.

[7]. Alibhai M, Balasundaram I, Bridle C. Cutaneous sinuses of dental origin. Journal of Surgical Case Reports, 8, $2012,6$. 